\title{
Integration of Spatial Processing in the WINNER B3G Air Interface Design
}

\author{
Martin Döttling ${ }^{1}$, Mikael Sternad ${ }^{2}$, Göran Klang ${ }^{3}$, Jörn von Häfen ${ }^{1}$, Magnus Olsson ${ }^{3}$ \\ 1: Siemens AG, München, Germany, E-mail: \{Martin.Doettling, Joern.von_Haefen\} @ siemens.com \\ 2. Signals and Systems, Uppsala University, Uppsala, Sweden, E-mail: Mikael.Sternad@signal.uu.se \\ 3: Ericsson AB, Stockholm, Sweden, E-mail: \{Goran.N.Klang, Magnus.A.Olsson\}@ericsson.com
}

\begin{abstract}
Numerous investigations of spatial processing algorithms are available in literature showing the benefit of MIMO systems in particular situations. From a system perspective, however, spatial processing has to provide adaptivity and scalability to a wide range of scenarios and has also to be seen in the context of its impact on other system services and functions, its enabling requirements, overhead, and robustness.
\end{abstract}

This paper summarizes recent work towards integrated spatial processing services and functions for an OFDM-based B3G air interface, developed in the European IST research project WINNER. The current status of the air interface and the multiuser spatial domain link adaptation concept is summarized. A baseline spatial scheme selection process is developed as part of the overall MAC radio resource control.

Keywords-WINNER, spatial processing, radio resource control, OFDM, MLMO

\section{INTRODUCTION}

The European research project WINNER is a cooperation of 38 partners from industry, operators, and academia which is partly funded by the European Union, with the overall goal to develop a single radio interface covering the full range from isolated hot spots to wide area cellular scenarios by using different modes of a common technology [1]. It targets increased data rates, low latency, and high system capacity based on adaptive transmission schemes, flexible spectrum usage, relaying, and advanced multi-antenna processing.

Spatial processing is a key enabler to meet these targets, since it provides an additional dimension of multiplexing, multiple access, and link adaptation. It also allows implementing enhanced (multi-user) interference management techniques. Depending on the specific needs of the data flows, robustness, coverage, or data rate can be increased and high overall spectral efficiency can be achieved. Due to the manifold usage scenarios to be accommodated, the WINNER multi-antenna architecture must be able to foster gains due to spatial diversity, spatial multiplexing, SDMA, beamforming, and spatial interference management in flexible combinations and with scalable amount of channel knowledge at the transmitter. We distinguish open-loop techniques, where no channel knowledge is available at the transmitter, from cases where we have a channel quality indicator (CQI, e.g., knowledge of SINR) or more complete channel state information (CSI, e.g. channel covariance matrix), either as long-term or short-term informa- tion. Such a scalable and adaptive concept is achieved by a multi-user spatial domain link adaptation concept, based on (linear) dispersion codes, beamforming, multi-user precoding, and per stream rate control (PSRC) [2].

The actual configuration and parameterization of the spatial processing is part of the MAC service radio resource control, which splits the available resources across cells and transport channels, and performs fast resource scheduling. The multiple functionalities of this service are interconnected and a joint optimization is not tractable. Even an iterative solution would compromise the quest for low latency. Therefore this paper outlines a baseline non-iterative procedure and split into functionalities that allows a flexible and channel-dependent resource assignment including spatial processing.

After a brief outline of the WINNER air interface in Section II, a generic processing architecture for the multiantenna processing is sketched in Section III. A simple baseline spatial scheme selection algorithm as part of the radio resource control is introduced in Section IV. Finally, conclusions are provided in Section V.

\section{THE WINNER AIR INTERFACE}

This section gives a brief outline of the current working assumptions (which of course still are subject to permanent changes and refinement in the ongoing project) for the WINNER air interface as far as they are related to spatial processing, see [3] for more details on the overall air interface. The WINNER air interface is a packet-oriented user-centric concept based on OFDM in downlink and Generalised Multi-Carrier (GMC) transmission (i.e. a generic frequency-domain signal generation with appropriate precoding) in uplink. FDD and TDD-based physical layer modes (PLM) are considered.

The basic resource and link adaptation units are called chunks, and consist of a contiguous set of $n_{s u b}$ subcarriers and $n_{s y m b}$ symbols, according to coherence bandwidth and time. In the FDD mode one chunk contains 96 symbols and spans $312.5 \mathrm{kHz} \times 345.6 \mu \mathrm{s}$. For the TDD mode each chunk contains 80 symbols ( $781.2 \mathrm{kHz} \times 108.8 \mu \mathrm{s}$ ). Spatial processing (e.g. by SDMA or spatial multiplexing) allows re-use of each timefrequency chunk, i.e. $Q_{c}$ chunk layers are available for chunk $c$ (cf. Figure 1). A frame of $691.2 \mu$ s duration contains 6 slots (configurable for UL/DL) for TDD and two slots for FDD. While the base stations operate in full duplex FDD mode, halfduplex FDD terminals are also supported. 
Resource scheduling and link adaptation is performed on a frame-basis. The overall resource partitioning between cells, beams, and traffic channels is done on a super-frame basis. A super-frame contains several frames in which the following data channels are transmitted along with their inband signaling:

- scheduled targeted data channels (TDC) for point-to-point communication,

- scheduled common data channels (CDC) for point-tomultipoint communication,

- contention-based direct access channels (DAC) for efficient use of resources, fast connection set-up, and peer-topeer communication.

Each super-frame also contains uplink and downlink synchronization channels, random access (RAC) and broadcast $(\mathrm{BCH})$ channels multiplexed in time. It is envisaged that the duration of a super-frame will be in the order of $5 \mathrm{~ms}$ to $10 \mathrm{~ms}$. Figure 2 shows an example containing 8 TDD frames.

The scheduling is partitioned into two levels, called service level control (SLC) and resource scheduling (RS). The service level controller has the overall responsibility for adjusting inter-flow fairness, assuring the fulfillment of service level contract agreements and total delay constraints. In general, it performs its task by assigning packets of flows for transmission to the resource scheduler taking into account QoS requirements. It may distribute the flows to several base stations that may utilize different WINNER modes. It works on a slower time scale than the resource scheduler.
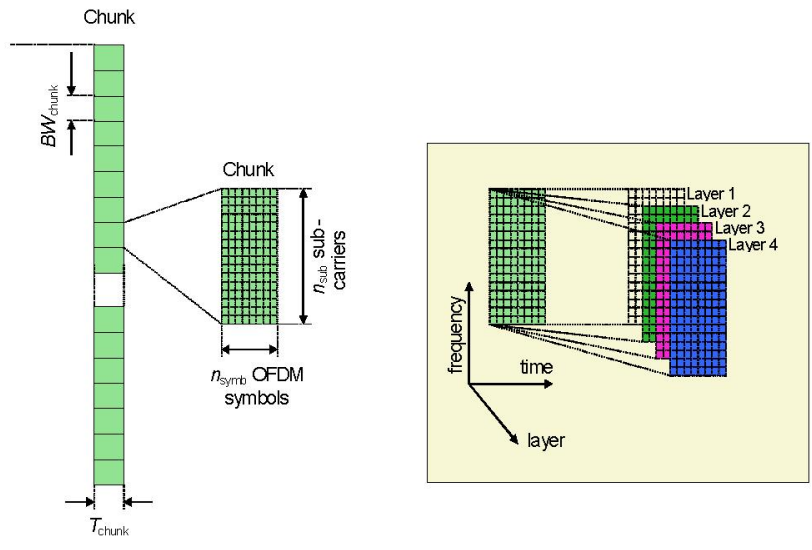

a)

Figure 1 Chunks and chunk layers

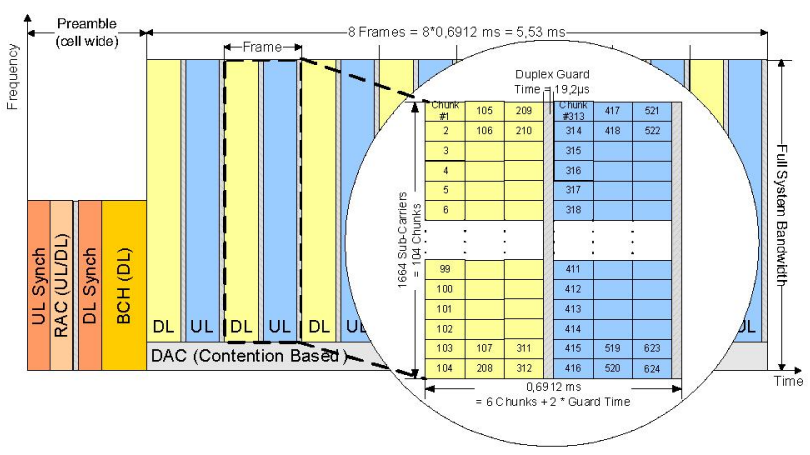

Figure 2 Super-frame structure
The resource scheduler optimizes the allocation of physical channel resources under constraints. Some constraints are inherent in the allocation scheme, others have different origins. These exogenous constraints are collected and updated by a constraint processing module. The constraints include chunk avoidance due to interference avoidance scheduling, chunk avoidance due to risk of high mutual interference, possibly subcarrier avoidance constraints due to shared spectrum use with other systems and the inter-operator partitioning of a shared WINNER spectrum.

\section{THE WINNER MULTI-ANTENNA CONCEPT}

In the following, a generic block diagram of the downlink transmitter processing is presented (for more details, see [2]).

Each flow (or subflow in case of PSRC) is channel encoded and interleaved, see Figure 3 . The channel encoded blocks are then mapped onto the chunk layers by the resource scheduler. The (maximum) number of layers in a chunk $c$, denoted $Q_{c}$, can be different for different chunks. Each layer carries data of one flow. For adaptive transmission it is envisioned that the scheduler will allocate chunks in a channel-dependent way, whereas for non-adaptive transmission the scheduler may multiplex the encoded bits onto chunks dispersed in (time and) frequency to benefit from diversity. Flows of different users can be multiplexed on arbitrary chunk layers. This enables SDMA per chunk, as well as OFDMA and TDMA.

After adaptive modulation, the symbols may be subject to non-linear precoding techniques, like Tomlinson-Harashima precoding (THP), or lattice coding techniques [2]. The precoded layers are then dispersed onto virtual antenna chunks with a dispersion code that provides the required amount of spatial diversity and spatial multiplexing. Typically, a linear dispersion code is used, but also non-linear schemes may be represented in the generic processing.

The virtual antenna chunk of each layer is then subject to power allocation and linear precoding. The term linear precoding in the present context covers techniques such as closed-loop transmit diversity, linear precoding and long-term beamforming, and also antenna or beam selection and hopping as well as random beamforming employed by opportunistic beamforming approaches. For cases with multi-user optimization, the linear precoding and power of virtual antenna chunks are optimized jointly. Finally, the antenna chunks are summed over the antennas and passed to OFDM modulation per antenna.

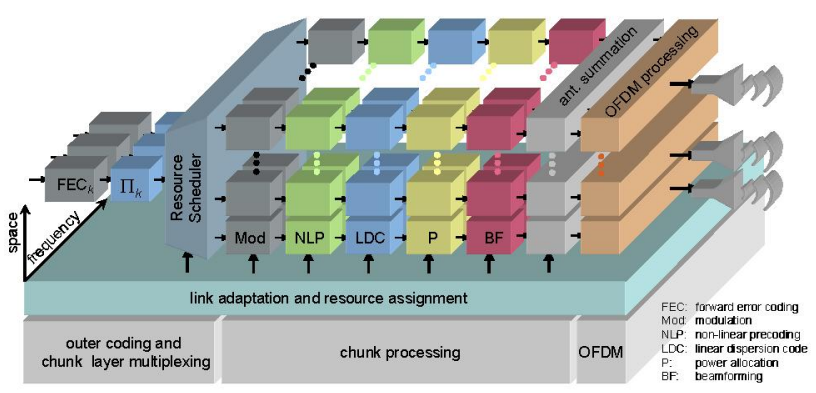

Figure 3 Generic downlink transmitter structure 
Depending on the scenario, system load, the propagation conditions, and the transport channel type (unicast, multicast or broadcast), varying spatial processing gains (like spatial multiplexing, spatial diversity, beamforming, and interference management) will be exploited to different degrees. Therefore, different spatial schemes will be applied. Typicall, in a particular spatial scheme not all function blocks of the generic framework will be operational.

\section{SPATIAL SCHEME SELECTION AND RADIO RESOURCE CONTROL}

Radio resource control involves the following basic steps and it operates on two time scales:

- with super-frame granularity:

- Resource partitioning: The distribution of the total radio resources to different transport channels is adjusted on this time scale, based on the aggregated demand within each transport channel. Guard chunks for interference avoidance scheduling are also defined and re-adjusted, to enable flexible spectrum use between WINNER operators/users and adaptive interference avoidance between neighbouring cells, sectors, and beams.

- Spatial scheme selection: Based on various decision criteria, the spatial processing algorithm for each flow is determined, i.e., the overall configuration of the physical layer blocks is determined.

- with frame granularity:

- Resource scheduling: The scheduled flows are allocated to space-time-frequency resources available within the super-frame.

- Link Adaptation including traditional and (multi-user) spatial link adaptation. In this step the actual parameters of the physical layer blocks are determined.

\section{A. Resource Partitioning}

Resource partitioning would in principle require knowledge of the capacity of each chunk realized by the actual subsequent resource allocation process, which depends on the flow allocated, the spatial scheme selected, and the link adaptation. In order to avoid highly complex and iterative algorithms, the resource partitioning can be based on evaluation of the aggregated capacity of all flows from the last superframes. From this, the average capacity per transport channel and radio resource can be estimated. A control loop can then adjust the resource partitioning to ensure that the requirements of each transport channel are met and account for all effects that determine the chunk capacity implicitly.

\section{B. Spatial Scheme Selection}

Spatial scheme selection per flow must be based on information and measurements available at the MAC layer. To reduce the complexity of the spatial scheme selection it is helpful to distinguish between different time scales of decisions. A temporal layering is proposed using static and long-term spatial scheme pre-configuration that acts as input and constraint for the spatial scheme selection.

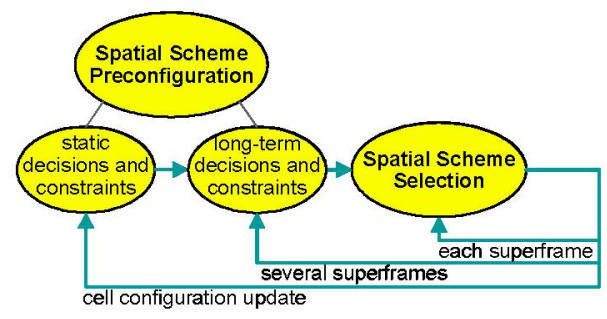

Figure 4 Spatial Scheme Preconfiguration and Selection

The static part of the spatial scheme pre-configuration evaluates all parameters, decisions and constraints that can only be changed with an update of the basic cell configuration. This includes the physical layer mode, type of deployment (cellular, isolated hot spot), cell range, and the base station (BS) antenna configuration. Each transport channel type has specific requirements and also the terminal capabilities that are fixed during the whole session. Therefore a static pre-selection of spatial schemes can be performed, e.g. by:

- consider beamforming only if BS antenna configuration allows it,

- $\quad$ prioritize beamforming and diversity for large cell ranges,

- consider multi-user precoding only for TDC,

- consider short-term adaptive precoding only for TDC in TDD mode,

- $\quad$ use a fixed spatial scheme for BCH, RAC.

Furthermore, mapping tables that indicate the possible spatial schemes for each transport channel and terminal capability, as well as a list of possible combinations of dispersion code, PSRC, and precoding type can be generated at this stage.

The long-term part of the spatial scheme pre-configuration evaluates all parameters, decisions and constraints that normally change on time scales slower than one super-frame. It also resolves issues that cannot be decided on a per-flow basis, like the overall selection of a scheme that applies to all users of a cell (e.g., configuration of a fixed beam approach, or multi-user precoding).

A slowly varying parameter of primary importance is the amount and quality of CQI/CSI information, which is typically related (amongst other) to the terminal velocity. This parameter governs the long-term pre-selection of applicable spatial schemes (e.g. long-term vs. short-term processing, type of precoding, etc. [4]) and also the overall transmission strategy (adaptive transmission vs. averaging, see Section IV C). Additionally the overall user distribution, the flows' QoS parameter, and long-term channel characteristics, like average SINR, long-term channel rank and eigenvalues are relevant input to this pre-selection process.

Important decision criteria that change on a short-term basis include, e.g., the number of active users, the cell load (due to packet-oriented transmissions), and the short-term channel characteristics. The spatial scheme selection considers these parameters and adapts the spatial scheme per flow within the pre-selection identified by the spatial scheme preconfiguration. An exemplary implementation includes the following major steps: 
- dispersion code selection based on the requirements regarding spatial multiplexing and diversity of the flow and the actual channel conditions,

- PSRC decision that determines whether segmentation of one flow will take place and the subflows will undergo individual link adaptation,

- precoding decision that first distinguishes between no beamforming, fixed beamforming and adaptive precoding and in the latter case further differentiates between short-term/long-term processing, whether multi-user optimisation is applied, and between linear and non-linear techniques.

The dispersion code selection determines the requested number of streams used for spatial multiplexing and diversity for each flow (Figure 5). This decision depends on the flow's requirements (service data rate, buffer content), transport channel type, terminal capabilities, and the opportunity of the propagation channel (e.g. channel rank, CQI). First degrees of freedom are allocated to spatial diversity as much as required for robustness (with the overall goal to minimize it). Then it is checked whether the QoS requirements for particular users would require spatial multiplexing and degrees of freedom are allocated accordingly (subject of course to the practical channel rank). Also here the goal is to minimize spatial multiplexing to leave sufficient possibilities to benefit from SDMA.

PSRC is a technique that can be selected for point-to-point flows that require spatial multiplexing in case only partial channel knowledge (CQI per stream) is available. If this option is used the flow is segmented into subflows that individually undergo channel coding and interleaving. These subflows are mapped to separate chunk layers.

The type of precoding or beamforming is determined to a large extent in the spatial scheme pre-configuration, since it depends on BS antenna configuration, deployment, cell range, transport channel type, user distribution and quality of channel knowledge. However, the number of users requesting resources and their buffer content is changing rapidly in a packet-oriented network. These parameters will also influence whether it is

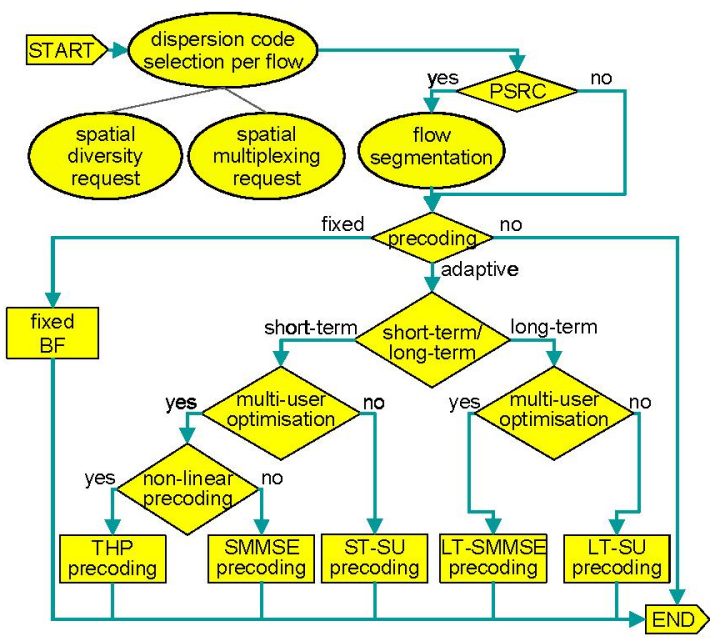

Figure 5 Spatial Scheme Selection Resource Scheduling worth configuring adaptive precoding with all its involved measurement and feedback overhead.

For adaptive processing we distinguish further between short-term (ST) and long-term (LT) processing, basically on static or long-term information used in the spatial scheme preconfiguration, such as PLM, user speed, and channel measurement quality. The latter, together with the number of active users, determines whether it is worth considering multi-user optimization.

As an example, we summarize the parameter set that would lead to the selection of short-term multi-user precoding, like THP or SMMSE (Successive Minimum Mean Square Error) based processing $[5,6]$. These techniques are primarily considered for TDC flows in the TDD mode, to benefit from reciprocity and avoid excessive feedback overhead. Furthermore, a sufficient number of active users with appropriate high buffer content and service data rate are required. Current investigations of the impact of channel estimation errors on such schemes [4] show that uplink pilots with $S I N R>20 \mathrm{~dB}$ and user speeds $\leq 3 \mathrm{~km} / \mathrm{h}$ are required to allow calculation of the precoding with an accuracy resulting in a maximum of $2 \mathrm{~dB}$ performance degradation if the channel estimator gain is in the order of $13 \mathrm{~dB}$ for linear precoding and of $17 \mathrm{~dB}$ for non-linear techniques. This must be ensured by appropriate channel estimation algorithms and/or by an additional pilot boost. Similarly, other criteria and parameter settings can be defined that lead to the decision for other spatial schemes.

\section{Resource Scheduling and Link Adaptation}

Within the resource scheduler, two basically different allocation strategies can be used:

- The adaptive transmission uses prediction of CQI to utilize the fast variations of the channel and of the interference for different terminals. The transmission parameters are adjusted to the frequency-selective channel of the selected user. The link adaptation may use combinations of adaptive modulation, coding adjusted to each chunk, and power control. The aim of the link adaptation is to provide a target SINR per bit for the FEC. Furthermore, by selecting individual chunks with adaptively adjusted rates tailored for each terminal, the throughput is increased and multi-user scheduling gains are realized. A combined TDMA/OFDMA/SDMA multiple access scheme is used. Adaptive transmission is feasible up to vehicular velocities, with the tight feedback loop made possible by the short frame length [7]. The CQI feedback overhead for adaptive transmission can be reduced to feasible limits by a combination of source coding that uses the frequency correlation and sub-sampling that uses the temporal correlation [7].

- Averaging strategies, on the other hand, are designed to combat and reduce the effect of the variability of the SINR, by e.g. interleaving, space-time coding and diversity combining. Non-frequency-adaptive transmission is required when fast channel state feedback is unreliable due to e.g. a high terminal velocity or a low SINR, or when the 
terminal does not support the adaptive transmission. Nonfrequency-adaptive transmission is also mostly required for point-to-multipoint communication belonging to the common data channel (CDC) flows. Non-frequency-adaptive transmission requires the $\mathrm{FEC}$ blocks to be mapped on a set of chunks that are widely dispersed in frequency or in different spatial channels to maximize diversity. The term non-frequency-adaptive transmission here also includes transmission that slowly adapts to the shadow fading, but averages over the frequency selective (fast) fading. Chunkbased TDMA/OFDMA would not provide sufficient diversity for small packets, therefore, either TDMA per OFDM symbol, OFDMA per subcarrier, or MC-CDMA are under consideration. All of them can be combined with an SDMA component.

A fundamental issue when designing such a resource scheduling and multiple access scheme, is how the scheduler should separate and group the users to realize SDMA. The simplest form of SDMA is traditional sectorisation, where the coverage area of a site is physically split into a number of sectors and the users in each sector are separated by time- and frequency resources. To derive the SDMA decision in a more general framework, the idea is to assign the different flows into different sets that include highly interfering flows within one set and guarantee an upper limit of interference across sets. Once such sets are established, we can use SDMA for flows of different sets, and other multiple access schemes (such as TDMA, FDMA) within one set [3].

Different basic algorithms apply depending on the amount of channel knowledge at the transmitter. If only a channel quality indicator (CQI) is available, so-called opportunistic or grid-of-beams beamforming approaches can be used. Here, sufficient interference suppression across beams is a priori given due to the proper design of the beams concurrently used. Therefore the pre-defined fixed beams are already the sets we are looking for.

If CSI is available at the transmitter and adaptive precoding is applied, the spatial sets can be generated by calculating the mutual correlation between users $[8,9]$. These methods are applied per chunk for frequency-adaptive flows and once over the whole allocation for non-frequency-adaptive flows. First the eigenvectors of all flows and the corresponding multi-user correlation matrix are calculated. For each flow, only the $k$ strongest eigenvectors should be used, according to the requested number of spatial streams (determined by the dispersion code selection or the PSRC decision). Then the flows are divided into groups with correlation higher than a pre-defined threshold. SDMA can now be performed for flows in different sets, whereas TDMA or FDMA has to be used for flows within one set.

Once these sets have been determined, the SDMA decision must be integrated in the overall resource allocation process, i.e. the decision must integrate the priorities due to QoS contracts and the opportunities and constraints due to the spatial channel characteristics. In particular it might be useful to revise the dispersion code selection (i.e. the requested number of spatial streams for spatial multiplexing and diversity per flow) in order to improve the opportunities for SDMA and to optimize the cell throughput. A successive allocation of the spatial degrees of freedom based on maximum increments of a target function is a promising candidate. Traditionally pure channel-dependent criteria have been used, such as maximum increments of sum-rate [10], however the target function should also take priorities into account.

\section{CONCLUSIONS}

This paper summarizes recent work towards integrated spatial processing services and functions for an OFDM-based B3G air interface, developed in the European IST research project WINNER. The current status of the air interface and the multi-user spatial domain link adaptation concept is summarized. A baseline spatial scheme selection process with temporal layering is developed as part of the overall MAC radio resource control. Relevant measurements and decision criteria for spatial scheme selection are discussed.

\section{ACKNOWLEDGMENT}

This work has been performed in the framework of the IST project IST-2003-507581 WINNER, which is partly funded by the European Union [and for Swiss partners the Swiss Government]. The authors would like to acknowledge the contributions of their colleagues.

\section{REFERENCES}

[1] WINNER, Wireless World Initiative New Radio, www.ist-winner.org

[2] M. Döttling, D. Astély, and M. Olsson, "A Multi-User Spatial Domain Link Adaptation Concept for Beyond 3G Systems," Proc.PIMRC 2005, Berlin, Germany, September 2005.

[3] IST-2003-507581 WINNER, "D2.10 Final report on identified RI key technologies, system concept, and their assessment", December, 2005.

[4] IST-2003-507581 WINNER, "D2.7 Assessment of Advanced Beamforming and MIMO Technologies", February 2005.

[5] D. Wang, E.A. Jorswieck, A. Sezgin, and E. Costa, "Joint TomlinsonHarashima precoding with orthogonal space time block codes for multiuser MIMO OFDM systems", in Proc. IEEE Vehicular Technology Conf. VTC 2005 Spring, Stockholm, Sweden, June 2005.

[6] V. Stankovic and M. Haardt, "Multi-user MIMO downlink precoding for users with multiple antennas", in Proc. of the 12th Meeting of the Wireless World Research Forum (WWRF), Toronto, ON, Canada, Nov. 2004.

[7] IST-2003-507581 WINNER, "D2.4 Assessment of adaptive transmission technologies", February, 2005.

[8] Del Galdo G., Haardt M., "Comparison of zero-forcing methods for downlink spatial multiplexing in realistic multi-user MIMO channels", in Proc. 59th IEEE Vehicular Technology Conf. (VTC 2004 Spring), (Milan, Italy), May 2004.

[9] Zhang Y.J., Letaief K.B., "An Efficient Resource-Allocation Scheme for Spatial Multiuser Access in MIMO/OFDM Systems", in IEEE Trans. Comm., vol. 53, no. 1, Jan. 2005, pp. 107-116.

[10] P. Tejera, W. Utschick, G. Bauch, and J.A. Nossek, "Sum-Rate Maximizing Decomposition Approaches for Multiuser MIMO-OFDM," Proc.PIMRC 2005, Berlin, Germany, September 2005. 